\title{
Optimizing Long-Term Treatment of Rheumatoid Arthritis with Systematic Documentation
}

\author{
K. M. Simonic, A. Holzinger, M. Bloice \\ Institute for Medical Informatics, \\ Statistics and Documentation \\ Medical University of Graz \\ Auenbruggerplatz 2, A-8036 Graz, Austria \\ klaus.simonic@medunigraz.at
}

\author{
J. Hermann \\ Division of Rheumatology and Immunology \\ Department of Internal Medicine \\ Medical University of Graz \\ Auenbruggerplatz 15, A-8036 Graz, Austria \\ josef.hermann@medunigraz.at
}

\begin{abstract}
About $1 \%$ of the population suffers from rheumatoid arthritis. They not only experience pain, but during the course of the disease their mobility is reduced due to a deterioration of their joints. To retard this destructive process an assortment of drugs are available today, however, for optimal results both medication and dosage have to be tailored for each individual patient. RCQM is a clinical information system that moderates this process: within the confines of the examination routine, physicians gather more than $\mathbf{1 0 0}$ clinical and functional parameters (time needed $<\mathbf{1 0}$ minutes). The amassed data are morphed into more useable information by applying scoring algorithms (e.g. Disease Activity Score (DAS), Health Assessment Questionnaire (HAQ)), which is subsequently interpreted as a function of time. The resulting DAS trends and patterns are ultimately used for treatment optimization and as a measure for the quality of patient outcome.

Graphical data acquisition and information visualization support the entire interaction between doctor and patient. Both are equally informed of the course of the disease and, in practice, treatment decisions are made jointly. The task of documentation becomes an integral part of the dialog with the patient. This yields an increased level of decision quality, higher compliance, and verifiable patient empowerment.
\end{abstract}

Index Terms-Clinical Information Systems, Decision Support, Patient Empowerment, Longitudinal Data Analysis

\section{The Clinical Picture Of Rheumatoid Arthritis}

The whole body is plagued with pains. They ravage the joints in particular, so that foot or hand or fingers cannot be moved even only slightly without eliciting cries of pain.

- Guillaume de Baillou, Liber de rheumatismo et pleuritide (1642)

Rheumatoid arthritis [1] is an inflammatory systemic disease that predominantly affects the joints. It is the most common form of arthritis, and has considerable social implications due to the costs incurred, the loss of productivity caused by the disease progression that may culminate in occupational disability, and the eventual need for assisted living. Rheumatoid arthritis has a prevalence of about $1 \%$ of the population, whereby women are $2 \frac{1}{2}$ times more commonly afflicted than men. The disease can develop at any time, but it occurs most often, and with increasing incidence, between the ages of 40 and 60 .
To this day, the etiology of rheumatoid arthritis is still not fully understood. The inflammation of the joint or, more precisely, of the synovial membrane, is a chronic, tissuespecific process to which a number of immune reactions contribute. The mucosa swells and proliferates, thereby gradually overgrowing and destroying the articular cartilage. Depending on the stage of the disease, the bony surface of the joint may become completely exposed. The destructive process often also affects the surrounding connective tissue including the bursa, tendons, and tendon sheaths, leading to deformation and functional disorders of the affected joints.

The clinical picture of rheumatoid arthritis varies with respect to the number and pattern of afflicted joints observed, possible involvement of internal organs, and the course of the disease. Disease onset is marked by early symptoms such as pain, morning stiffness, and swelling of peripheral joints that usually develop gradually over a period of a few weeks. After several months, the first changes in the affected structures appear. Magnetic resonance tomography begins to show alterations in the synovial membrane, occasionally in combination with osseous edema and, within the first 2 years, radiographic evidence is present for $70 \%$ of all patients. By the time the physical deformations are recognized, severe, irreversible joint damage has already occurred; concomitant malaise and depression are now frequent.

Rheumatoid arthritis is incurable but today a variety of medications are available to delay the progression of the disease [2]. Disease Modifying Antirheumatic Drugs (DMARDs) are used as basic treatment and their administration must be continually adjusted according to the patient's individual situation. In the event of an unsatisfactory response to therapy, or the loss of drug efficacy, the basic treatment is escalated to include biologicals, i.e. medications produced by biological engineering that specifically block receptors or signal molecules (biological response modifiers). This multi-tiered approach requires a stringent monitoring of disease activity and its development. This is where information management comes in. 


\section{Scores For The Evaluation Of Disease Activity}

Since rheumatoid arthritis is a systemic disease, the precise evaluation of disease activity depends on a number of findings:

- Clinical status of the joints, with special attention paid to swelling and tenderness [3].

- Laboratory parameters: erythrocyte sedimentation rate (ESR) and C-reactive protein (CRP).

- Global assessment of the disease by both physician and patient, using a visual analogue scale.

These findings, taken together, determine the Disease Activity Score (DAS), a standardized and validated measure of disease activity for rheumatoid arthritis [4], [5]. Depending on the number of joints assessed and included in the formula, the results are categorized as belonging to either DAS28, DAS32, DAS44 or DAS68. International benchmarks and/or clinical studies are normally based on DAS28, for the very reason that considerable time and effort is required for the examination.

With these facts in mind, how do the clinical parameters of inflammation correlate with joint damage caused by the disease? Welsing et al. [6] answer this question by comparing the changes in disease activity (as represented by DAS28) with structural changes seen in x-ray images. While a DAS28 $\geq 5.1$ indicates that joint destruction is proceeding unabated, when disease activity is low enough, for long enough, as reflected by a DAS $28<3.2$, destructive activity is virtually stopped. In the range between these two limits $(3.2 \leq$ DAS2 $<<5.1)$ and with a falling score, the course of the disease slows and its consequences diminish, although this relationship is individually variable and nonlinear. Furthermore, Fransen et al. [7] proved that, by utilizing the disease activity scoring system, patient outcome is considerably improved.

Functional impairment and the reduced mobility of patients are assessed using the Health Assessment Questionnaire (HAQ). The HAQ index is an internationally recognized measure of the functional capacity of individuals and reflects everyday activities such as getting up, dressing, eating, walking, personal hygiene, etc. The higher the HAQ index, the greater the degree of disability.

\section{Systematic Documentation For The OPTIMIZATION OF TREATMENT}

It would be tempting to believe that, by using scores, the management of patients with rheumatoid arthritis is a simple task. However, this is in fact not the case. Rheumatologists complain that the many clinical and functional parameters can only be collected and documented in the context of clinical trials. "In daily routine, dedicating one hour per patient is simply not feasible!" is a typical response. "The entire visit may last no longer than 15 minutes!" The RCQM clinical information system described in this section is the result of the joint efforts of the Institute for Medical Informatics, Statistics and Documentation, and the Division of Rheumatology and Immunology at the Medical University of Graz to develop a documentation tool that fulfills these time constraints and other requirements.

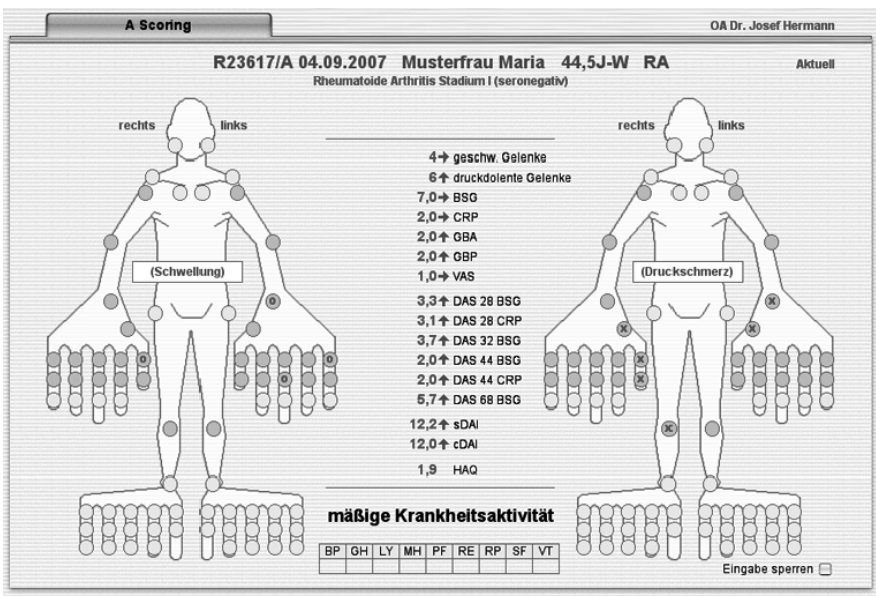

Fig. 1. Screenshot of the interactive graphical representation of the status of the patient's joint. Using nothing but the mouse, swollen and tender joints are marked (left and right schema respectively). In the middle of the screen an up-to-date score list of the current status of the patient is displayed.

RCQM is an application that manages the flow of data and information in a rheumatology outpatient clinic on the basis of a quality management process model. All the clinical and functional parameters required for complete evaluation of the patient are collected in a standardized form via an interactive graphical representation of the human body, as shown in Figure 1. The interface represents all 72 joints that are palpated for swelling and tenderness. In general, the physician performs the examination following a mental model based on structural groups (fingers, toes, large joints) and symmetry. RCQM allows for documentation to be quickly performed by taking this mental model into account and implements it to guide the input procedure in a context-specific manner, while not stipulating any particular method. The data acquired are used to calculate a number of scores; together with all previous findings, these constitute the information basis for analysis and evaluation of disease activity.

The second main requirement of RCQM was to provide decision support with regard to the optimization of treatment strategy. This therapy is usually administered over the long term, typically spanning decades. The medical goals of this treatment may be summarized as follows:

- Prevention of disease progression and functional deterioration.

- Absence of swollen joints.

- Normalization of inflammation parameters.

- Freedom from pain and/or complaints.

By translating these goals into the context of the functionality of RCQM, the following standard operating procedures can be defined:

- High disease activity (DAS28 $\geq 5.1$ ): This represents acute danger of damage to the joints. The treatment goal is to reduce disease activity to moderate values as quickly as possible with a high dosage of DMARDs and supplemental biologicals. Patients should be monitored every $1-2$ weeks. 


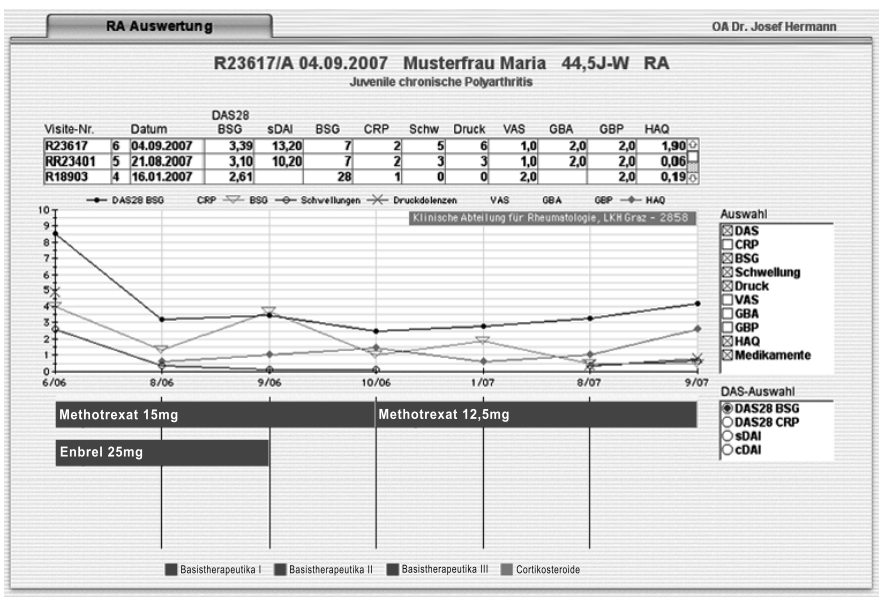

Fig. 2. Screenshot of the temporal course of disease activity combined with the current medication and dosage. Upon presentation, the patient exhibited high disease activity (the DAS is represented by the uppermost line). Following a treatment with Methotrexate 15mg (DMARD) and Enbrel $25 \mathrm{mg}$ (biological agent) the disease activity was dramatically reduced. However, while continuing with this administration, no further decrease of the DAS was observed, and therefore Enbrel was discontinued. Due to the fact that DMARDs require several weeks to months for the intended effect to be observeable, it was not until the fourth visit that the DAS registered 2.6. With the score now in the lowest group, the treatment strategy shifted to dosage minimization (Methotrexate $12.5 \mathrm{mg}$ ). Scores slightly increased but remained below the group threshold. On the current visit, disease activity has risen above 3.2 and counter measures are being taken by increasing the Methotrexate dosage to $15 \mathrm{mg}$. The next visit is scheduled for 2 months' time.

- Moderate disease activity: $(3.2 \leq$ DAS28 < 5.1): The aim is to reduce the score with an individually optimized/balanced mono-therapy or combined therapy with different DMARDs and optional biologicals, with respect to the course of disease, swelling, pain, adverse effects, and tolerance. Patients should be monitored at intervals of 1-3 months.

- Low disease activity (DAS28 < 3.2): This represents the endpoint of the disease activity minimization procedure. The aim here is to retain the status with an optimized/minimized administration of medications. Checkups should be scheduled for every 3 months.

In other words, for any DAS28 $\geq 3.2$, the primary goal is the reduction of disease activity; for all other scores, the aim of the treatment is to find the lowest maintenance dosage to preserve the patient's low disease activity status in order to reduce the risk of adverse effects and to prevent any subsequent loss of efficacy. Typically, this strategy leads to scores near the upper limit of acceptability. It must be borne in mind that, while the procedural model is static, the treatment is tailored for every individual patient and varies with time.

RCQM supports the decision process by aggregating the entities in the aforementioned information base with respect to time. The resulting time series reveals patterns and trends that, when visualized in combination with the medication, permit a previously unknown form of data analysis (see Figure 2). The interpretation of the data as a function of time gives the rheumatologist decisive advantages, i.e.
- the execution of division-of-labor processes without loss of quality,

- retrospective evaluation of the administered treatment (effectiveness check),

- differential-diagnostic evaluation of the current situation,

- individual optimization of the use of medications in the context of the defined treatment goals.

Further functions of RCQM are the semi-automatic generation of medical reports and the documentation of services. RCQM also assists communication between doctor and patient.

\section{PATIENT EMPOWERMENT}

Anyone who spends a lifetime fighting against the sequelae of rheumatoid arthritis needs knowledge about the disease in general and, in particular, information about their individual condition. Patients should, therefore, be regularly informed about their current status. However, in practice the tight schedule of a hospital outpatient department and the fact that modern clinical findings are not easily communicable hinders the dialog between doctor and patient. RCQM makes it possible to bridge this information gap by providing a patientspecific view of the data stored in the information base.

At the division of rheumatology, each examination room contains a computer for the physician. In addition, a large LCD television is mounted on the wall and displays the patient's view of the RCQM application. During the visit, the patient can follow the doctor's input, ask questions about the chronological course of disease activity and medication, gain clarification, establish the facts, and understand their current health situation. RCQM uses the same intuitive graphical elements for the information presentation (joints schema, charts, etc.) for both the doctor and the patient and thus, in the truest sense of the term, provides a common basis for discussion. The act of documentation therefore becomes an integral part of the communication between doctor and patient. When the doctor interrupts the examination to enter data, the patient does not feel excluded from the process. What the patient does not see on the screen are the hard to understand medical documents meant only for experts. To our knowledge, there is no other medical information system that comes even close to offering comparable functionality.

The situation described above has consequences far beyond the changes in the consultation procedure. The patients take a much more active role in their treatment decisions. Subsequently, increased decision quality and sustainable improved compliance are achieved, both important factors for high quality results. In fact, the more complex the medical procedure, the greater the impact on quality.

\section{QUALITY SCRUTINIZED}

To evaluate RCQM, an analysis was made on the progress of disease activity for all documented patients of both sexes who were first examined before 2006 and received at least 5 follow-up examinations. The time frame between followups was at most 12 months. This set of patients $(n=175)$, 


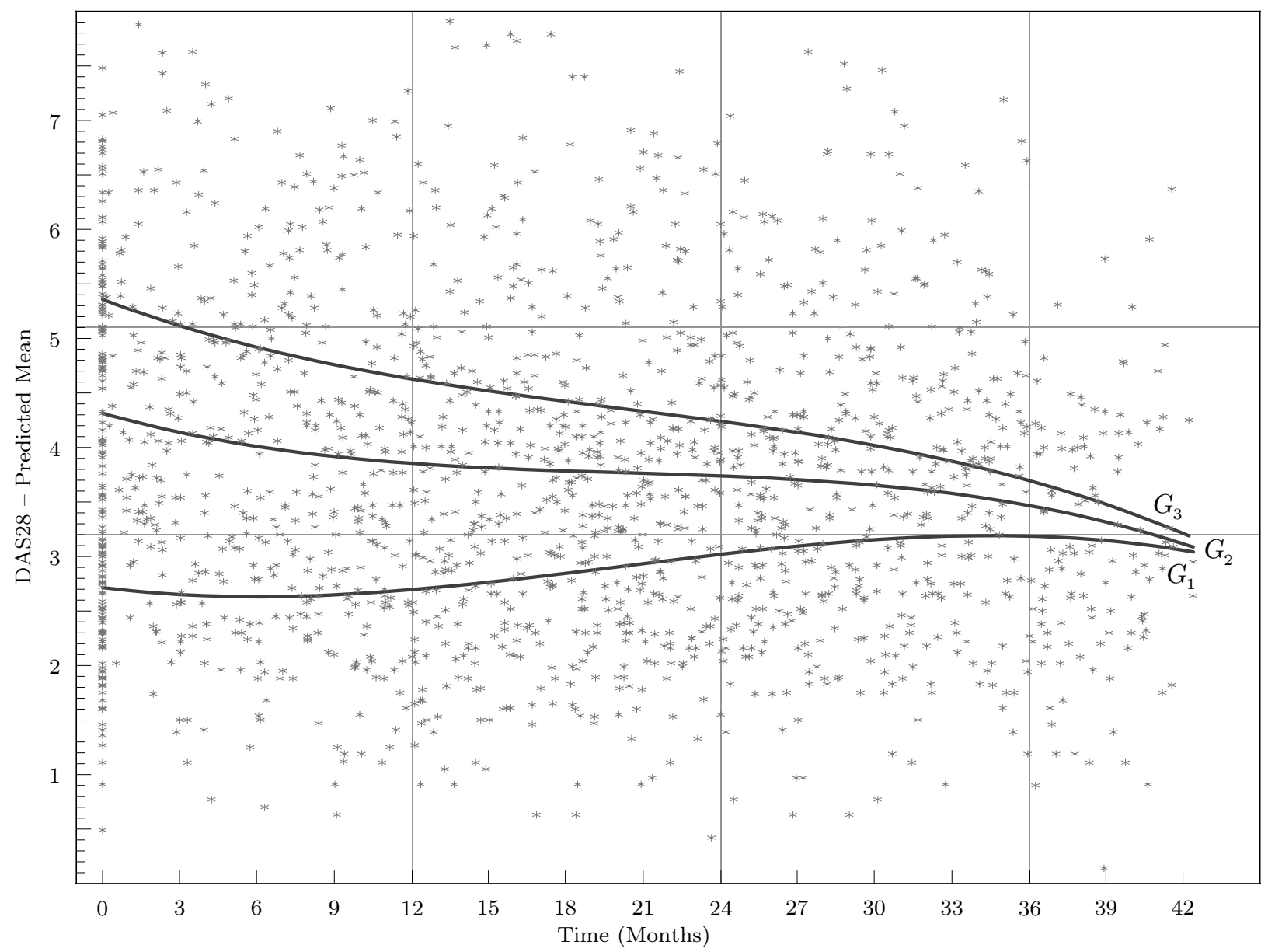

Fig. 3. DAS28 predicted mean responses for patients with initial low $\left(G_{1}\right)$, moderate $\left(G_{2}\right)$ and high $\left(G_{3}\right)$ disease activities, analyzed over a period of 42 months. The graph clearly shows the different therapy goals: for groups $G_{2}$ and $G_{3}$ (DAS28 $\geq 3.2$ ), the curves are monotonically non-increasing. Although a patient's course of disease activity typically develops in downward trending waves, the predicted mean of the scores suppress these individual fluctuations. For group $G_{1}$, the wavelike form remains visible. This is due to the shifts in treatment strategy; however, as expected the curve's inflection point falls into the upper limit of the low group. Furthermore, the graph reveals that, on average, all groups reach the desired endpoint; patients with a high DAS were led out of the acute danger zone within 4 months. The data set consists of 175 patients who made a total of 1782 visits.

which resulted in a total of 1782 visits (min.: 6, max.: 27, median: 11 visits/patient), was divided into three groups depending on the initial disease activity upon first examination. Group $G_{1}$ constituted 76 patients with low disease activity (DAS28 $<3.2$ ); group $G_{2}$ comprised 55 patients with moderate disease activity $(3.2 \leq$ DAS $28<5.1)$; the remaining 44 patients were assigned to group $G_{3}$ as they presented an initial high score (DAS28 $\geq 5.1$ ). Of interest was, to what degree the DAS28 value changed for each of the 3 groups of patients over a period of $3 \frac{1 / 2}{2}$ years.

Longitudinal data present the problem that two aspects of the data require modeling: the mean response over time and, as these data are correlated, the covariance (the time dependence between the repeated measures) obtained for a particular individual. To determine the DAS28 mean response over time, a linear mixed effects model for longitudinal data

$$
y=\boldsymbol{X} \beta+\boldsymbol{U} \gamma+\varepsilon
$$

was formulated [8]. Linear mixed effects models have the distinctive feature that the mean response can be modeled as a combination of population dependent parameters, which are assumed to be shared by all individuals, and subjectspecific effects unique to a particular individual. The former are referred to as fixed effects, while the latter are termed random effects, as they can vary randomly from one individual to another, thereby accounting for sources of natural heterogeneity in the population. In model (1), $\beta$ denotes the vector of fixed effects present: a time effect that describes the change of the regression parameters with time $t$; further a group effect that represents the intercept per group $G_{i}, i=1,2,3$; as well as an interaction term $t G_{i}$ between time and group. The random effects, $\gamma$, that are taken into consideration allow the intercept and slope between the patients to be varied at random. $\boldsymbol{X}$ and $\boldsymbol{U}$ denote the corresponding design matrices; $\varepsilon$ represents the error term.

The number of repeated observations per patient, and their irregular timing (a problem frequently encountered in the evaluation of procedures in medical practice), allows for, but 
TABLE I

Solution Of The Linear MiXed Model For The FiXed Effects

\begin{tabular}{|c|c|c|}
\hline Effect & Estimated Parameter (S. E.) & $\boldsymbol{p}$-Value \\
\hline Intercept & $2.7146(0.1031)$ & $<0.0001$ \\
\hline$t$ & $-0.02977(0.01662)$ & 0.0750 \\
\hline$G_{1}$ & 0 &. \\
\hline$G_{2}$ & $1.5986(0.1496)$ & $<0.0001$ \\
\hline$G_{3}$ & $2.6441(0.1528)$ & $<0.0001$ \\
\hline$t G_{1}$ & 0 &. \\
\hline$t G_{2}$ & $-0.03662(0.006449)$ & $<0.0001$ \\
\hline$t G_{3}$ & $-0.05927(0.006971)$ & $<0.0001$ \\
\hline$t^{2}$ & $0.002923(0.000990)$ & 0.0036 \\
\hline$t^{3}$ & $-0.00005(0.000017)$ & 0.0053 \\
\hline Residual & $0.9180(0.03555)$ & $<0.0001$ \\
\hline
\end{tabular}

TABLE II

COVARIANCE MATRIX OF THE RANDOM EFFECTS

\begin{tabular}{|c|c|c|c|}
\hline Effect & \multicolumn{3}{|c|}{ Matrix Entries } \\
\hline Intercept & 0.2170 & 0.010010 & -0.00029 \\
\hline$t$ & 0.010010 & 0.005899 & -0.00015 \\
\hline$t^{2}$ & -0.00029 & -0.00015 & 0.00000392 \\
\hline
\end{tabular}

also requires, the introduction of additional structure on the underlying mean response trend. One widely adopted approach is to describe the patterns of change in terms of polynoms, thereby modeling the means as an explicit function of time. Polynomials were introduced into both the fixed and the random effects. Here the fixed cubic time effect was significant ( $p=0.0053$ ). Fourth-order polynomials were tested but did not have a significant effect on the result. For the random effects, only the quadratic term was considered, as the model no longer converged for higher-order polynomials. All model assumptions were tested with residual analyses. SAS 9.1's MIXED procedure was used for the analysis, the results are given in Tables I and II.

The visualization of the predicted DAS28 mean response trends for the different groups is illustrated in Figure 3. The analysis shows a tendency toward stagnation in the group with low disease activity, while the average scores in the other two groups dropped enough to fall into the range of low disease activity. In other words, disease progress was considerably slowed and, assuming a continuous trend, the patients have a good chance of retaining their physical capabilities well into old age.

\section{CONCLUSION}

RCQM borrows from methods and techniques that, due to the high efforts involved, have until now only been used in clinical trials, and applies them to the highly time constrained environment of the daily clinical routine. It represents a new generation of clinical information systems, which collects all treatment-relevant data quickly and in a quality processoriented manner and condenses them into information as a function of time. The physicians use these patterns and trends to make decisions in order to meet predefined treatment goals and to provide optimal medication and dosage tailored to the individual. The result is excellent quality of care combined with improved efficiency, both of which benefit patients with rheumatoid arthritis. They have a good chance of retaining their physical capabilities well into old age. In turn, this preservation of capability is of benefit to general society, from both economic and social points of view. In short, RCQM creates an all-win situation.

\section{ACKNOWLEDGMENT}

The authors would like to thank Univ.-Prof. Dr. Winfried Graninger, Head of the Division of Rheumatology and Immunology, Medical University of Graz, and President of the Austrian Society for Rheumatology and Rehabilitation for many years of cooperation.

\section{REFERENCES}

[1] D. M. Lee and M. E. Weinblatt, "Rheumatoid arthritis," Lancet, vol. 358, no. 9285, pp. 903-11, Sep 2001. [Online]. Available: http://linkinghub.elsevier.com/retrieve/pii/S0140673601060755

[2] J. S. Smolen, R. Landewé, F. C. Breedveld, M. Dougados, P. Emery, C. Gaujoux-Viala, S. Gorter, R. Knevel, J. Nam, M. Schoels, D. Aletaha, M. Buch, L. Gossec, T. Huizinga, J. W. J. W. Bijlsma, G. Burmester, B. Combe, M. Cutolo, C. Gabay, J. Gomez-Reino, M. Kouloumas, T. K. Kvien, E. Martin-Mola, I. McInnes, K. Pavelka, P. van Riel, M. Scholte, D. L. Scott, T. Sokka, G. Valesini, R. van Vollenhoven, K. L. Winthrop, J. Wong, A. Zink, and D. van der Heijde, "Eular recommendations for the management of rheumatoid arthritis with synthetic and biological diseasemodifying antirheumatic drugs," Ann Rheum Dis, vol. 69, no. 6, pp. 964 75, Jun 2010.

[3] P. L. C. M. van Riel, J. Fransen, and D. L. Scott, Eular handbook of clinical assessments in rheumatoid arthritis, $3^{\text {rd }}$ ed. Alphen/Rijn, NL: Boeken, 2004.

[4] D. M. van der Heijde, M. A. van 't Hof, P. L. van Riel, L. A. Theunisse, E. W. Lubberts, M. A. van Leeuwen, M. H. van Rijswijk, and L. B. van de Putte, "Judging disease activity in clinical practice in rheumatoid arthritis: first step in the development of a disease activity score," Ann Rheum Dis, vol. 49, no. 11, pp. 916-20, Nov 1990.

[5] M. L. Prevoo, M. A. van 't Hof, H. H. Kuper, M. A. van Leeuwen, L. B. van de Putte, and P. L. van Riel, "Modified disease activity scores that include twenty-eight-joint counts. development and validation in a prospective longitudinal study of patients with rheumatoid arthritis," Arthritis Rheum, vol. 38, no. 1, pp. 44-8, Jan 1995.

[6] P. M. J. Welsing, R. B. M. Landewé, P. L. C. M. van Riel, M. Boers, A. M. van Gestel, S. van der Linden, H. L. Swinkels, and D. M. F. M. van der Heijde, "The relationship between disease activity and radiologic progression in patients with rheumatoid arthritis: a longitudinal analysis," Arthritis Rheum, vol. 50, no. 7, pp. 2082-93, Jul 2004.

[7] J. Fransen, H. B. Moens, I. Speyer, and P. L. C. M. van Riel, "Effectiveness of systematic monitoring of rheumatoid arthritis disease activity in daily practice: a multicentre, cluster randomised controlled trial," Ann Rheum Dis, vol. 64, no. 9, pp. 1294-8, Sep 2005.

[8] G. M. Fitzmaurice, N. M. Laird, and J. H. Ware, Applied Longitudinal Analysis, ser. Wiley Series in Probability and Statistics. Hoboken, NJ: Wiley, 2004. 